Internist 2015 $\cdot 56: 716$

DOI 10.1007/s00108-014-3642-3

Online publiziert: 9. April 2015

(c) Springer-Verlag Berlin Heidelberg 2015

J. Hübner · M.R. Noftz $\cdot$ M. Schnoor $\cdot$ A. Katalinic

Institut für Sozialmedizin und Epidemiologie, Universität zu Lübeck

\title{
Erratum zu: Screening auf Kolorektal-, Haut-, Brust- und Prostatakrebs. Basiswissen für die Beratung
}

Im o. g. Beitrag (Ausgabe 10/2014; Rubrik CME) haben wir, die Autoren, auf S. 1175 ausgeführt, dass falsch positive Befunde bei der Früherkennungskoloskopie (einschließlich Abklärungsdiagnostik) in maximal 5\% der Fälle vorkommen. Diese Obergrenze ist von einigen klinisch tätigen Kollegen als zu hoch kritisiert worden. Wir haben dies zum Anlass genommen, nochmals die Literatur kritisch zu prüfen. Leider wird über die Spezifität der Koloskopie nur in wenigen Studien und dann eher narrativ berichtet, was sicher auch durch methodische Schwierigkeiten bedingt ist. In Einzelfällen kommen Fehlklassifikationen unvermeidbar vor, da das Ergebnis der pathologischen Beurteilung nicht immer eindeutig ist [1]. In einer systematischen Übersichtsarbeit wird die Spezifität der Früherkennungskoloskopie mit 99,8\% angegeben [2]. Wir korrigieren unsere Angabe nun dahingehend, dass mit falsch positiven Befunden in weniger als $1 \%$ der Untersuchungen zu rech- nen ist. Für die konstruktiven Hinweise der Kliniker bedanken wir uns.

Die richtige Antwort der zu dieser Aussage gestellten CME-Frage ist dadurch nicht berührt.

\section{Korrespondenzadresse}

\section{Prof. Dr. A. Katalinic}

Institut für Sozialmedizin und Epidemiologie, Universität zu Lübeck

Ratzeburger Allee 160, 23562 Lübeck

Alexander.Katalinic@uksh.de

\section{Literatur}

1. Sheu L, Gottenborg EW (2014) False-positive results from a diagnostic colonoscopy: a teachable moment. JAMA Intern Med 174(5):665-666

2. Allameh Z, Davari M, Emami MH (2011) Sensitivity and specificity of colorectal cancer mass screening methods: a systematic review of the literature. Iran J Cancer Prev 4(2):88-105

Die Online-Version des Originalartikels können Sie unter http://dx.doi.org/10.1007/s00108-0143601-z finden. 